\title{
Deterioro de Generadores de Alta Tensión Ocasionado por la Separación de la Pintura Conductora en las Bobinas del Estator
}

\author{
Alejandro E. González, Arturo García e Ignacio R. Campuzano \\ Instituto Nacional de Electricidad y Energías Limpias, Reforma 113, Col Palmira, Cuernavaca, Morelos, CP \\ 62490, México, (e-mail; agv@iie.org.mx; tevillo@iie.org.mx; rcm@iie.org.mx)
}

Recibido Nov. 18, 2015; Aceptado Ene. 20, 2016; Versión final Mar. 15, 2016, Publicado Oct. 2016

\begin{abstract}
Resumen
En este artículo, se presenta el deterioro de un generador que sufrió un sobrecalentamiento de $150{ }^{\circ} \mathrm{C}$. El esfuerzo térmico ocasionó la separación de la pintura conductora de la superficie del aislamiento, en algunas zonas del devanado, debido a que la pintura conductora no cumplió con los requerimientos de la Clase F. Esto generó cavidades internas debajo de la pintura conductora, las cuales ocasionaron pulsos esporádicos de descargas parciales de gran magnitud a bajos niveles de la tensión de prueba. Este mecanismo fue monitoreado a través del tiempo. Las descargas parciales fueron erosionando la pintura conductora, dejando zonas eléctricamente flotadas, en la superficie de las bobinas. Finalmente, las descargas parciales erosionaron el sistema aislante, ocasionando una falla a tierra en un período de doce años. El objetivo es aportar esta experiencia al acervo técnico relacionado con los mecanismos de deterioro del sistema aislante de los generadores de alta tensión.
\end{abstract}

\section{Deterioration of High Voltage Generators Caused by the Separation of the Conductive Paint in the Stator Coils}

\begin{abstract}
In this paper, the deterioration of a generator that suffered an overheating of $150{ }^{\circ} \mathrm{C}$ is presented. The thermal stress caused the separating of the conductive paint from the surface of the insulation, in some areas of the winding, because of the conductive paint did not meet the requirements of the Class $\mathrm{F}$. This caused internal cavities under the conductive paint, which generated great magnitude sporadic pulses of partial discharges at low test voltage. This mechanism was monitored through the time. Partial discharges were eroding to the conductive paint, leaving ungrounded areas on the surface of the coils. Finally, partial discharges eroded to the insulation system, causing a failure to ground over a period of twelve years. The objective is to provide this experience to the present technical knowledge about deterioration mechanisms of high voltage generator insulation systems.
\end{abstract}

Keywords: high voltage generators; conductive paint; stator coils; overheating 


\section{INTRODUCCIÓN}

La confiabilidad de un generador de alta tensión, depende en gran medida, de las condiciones dieléctricas del devanado del estator. En consecuencia, cuando un generador es expuesto a un sobrecalentamiento, es necesario efectuar el diagnóstico de su sistema aislante. Existen diversas técnicas para efectuar el diagnóstico de un generador. Las normas internacionales han ido regularizando los métodos de prueba. Sin embargo, son las experiencias publicadas, acerca de la evaluación de los sistemas aislantes, las que han ido enriqueciendo la capacidad de detección de los mecanismos de deterioro en forma incipiente (Dorrbercker, et al., 2012; Darwanto, et al., 2012; Torres, et al., 2010).

El sobrecalentamiento de un generador se puede generar por sobre carga, por altas corrientes de falla, o por fallas en el sistema de enfriamiento. En este artículo, se presenta el análisis de un generador que sufrió un sobrecalentamiento durante su puesta en servicio, después de ser sometido a un proceso de mantenimiento. Las características del generador son las siguientes: $32 \mathrm{MVA} / 13.8 \mathrm{kV}$, enfriado con aire forzado en ciclo cerrado, con sistema aislante Clase $\mathrm{F}$ a base de mica, fibra de vidrio y resina epóxica, con turbina de vapor, fabricado en 1975 y con una antigüedad en servicio de 18 años.

El sistema de enfriamiento del generador no operó correctamente durante la puesta en servicio. Los sensores de temperatura registraron del orden de $150^{\circ} \mathrm{C}$, en la superficie de las bobinas del estator, por lo que el generador fue retirado de servicio. Todo el proceso de arranque, sincronización, toma de carga y salida de servicio del generador, tuvo una duración del orden de 1 hora. Sin embargo, no se logró precisar el tiempo real en que los devanados estuvieron sometidos al sobrecalentamiento.

De acuerdo con la literatura especializada, un sobrecalentamiento puede generar problemas de delaminación y/o daños en la pintura conductora (IEEE 1310, 2012). La delaminación se genera porque el conductor se calienta y se expande más rápidamente con respecto al sistema aislante. Asimismo, el conductor se contrae y se enfría más rápidamente que el aislamiento. Esto genera esfuerzos mecánicos que tienden a separar el aislamiento de la superficie del conductor, tal como se muestra en la Fig. 1. Este mecanismo genera un patrón de descargas parciales (DP), donde los pulsos positivos son mayores con respecto a los pulsos negativos, en la forma de onda sinusoidal de medición y calibración del detector de DP (Hudon y Bélec, 2005). La delaminación también puede ocasionar la separación generalizada entre las capas aislantes, incrementando considerablemente la cantidad de cavidades internas (Hudon y Bélec, 2005). Por lo tanto, un devanado con problemas de delaminación presenta incrementos considerables de DP y tangente de pérdidas (tan 8), en función de la tensión de prueba (Znidarich, 2008; Sathiyasekar, 2009).

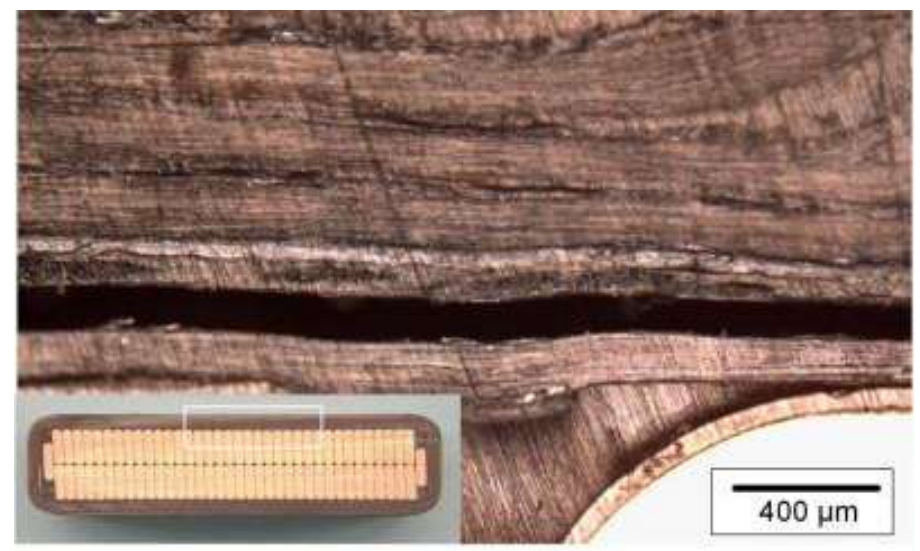

Fig. 1 Vista amplificada de la separación del aislamiento del conductor de una bobina.

Con respecto a la pintura conductora, los esfuerzos térmicos pueden modificar su resistencia superficial, la cual varía en el intervalo de 0.1 a $10 \mathrm{k} \Omega$ por cuadro (Azizi y Gholami, 2014). Este valor se puede incrementar por la oxidación de las partículas de carbón a temperaturas elevadas (Stone, et al, 2004). En estas condiciones, las bobinas ya no adquieren correctamente el potencial de tierra, al ponerse en contacto con el núcleo del estator, por lo que se generan DP a la ranura (Stone, et al, 2008). Este fenómeno genera un patrón de DP, donde los pulsos negativos son mayores a los positivos (Hudon y Bélec, 2005). Por otro lado, la actividad de DP a la ranura y la abrasión de la pintura conductora, por la vibración de las bobinas en las ranuras del estator, ocasionan la formación de zonas sin pintura conductora, con el aislamiento erosionado, tal como se muestra en la Fig. 2. Tanto la delaminación como el fenómeno de DP a la ranura generan altos niveles de DP (Hudon y Bélec, 2005), por lo que constituyen un alto riesgo para el aislamiento (Azuaje y Torres, 2004; Stone, et al, 2008; Hudon y Belec, 2005). 


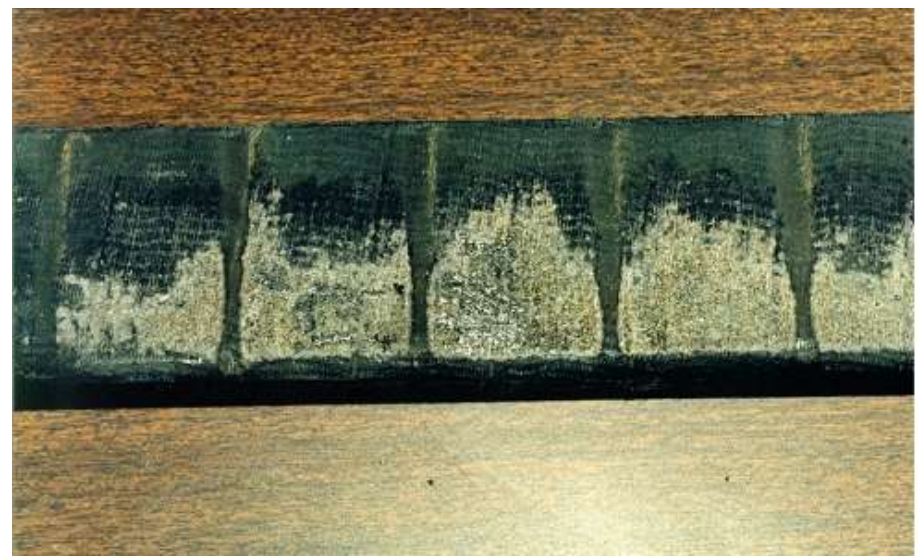

Fig. 2 Deterioro por descargas a la ranura, ocasionado por la abrasión de la pintura conductora.

El deterioro de la pintura conductora, también puede afectar la zona de traslape entre ésta y la pintura graduadora, ocasionando problemas de graduación de campo eléctrico. Se generan DP externas visibles, con niveles mayores de 10 nC (Dorrbercker, et al, 2102; Campuzano, 2016; Krpal and Mráz, 2014). Una evidencia de este fenómeno es el polvo blanco en la zona de traslape de las pinturas, el cual resulta de la erosión del aislamiento por la acción de las DP, ver la Fig. 3. En el caso del generador bajo estudio, inmediatamente después del sobrecalentamiento, se llevó a cabo su evaluación dieléctrica. Se registraron pulsos esporádicos de DP de gran magnitud, a bajos niveles de la tensión de prueba. Sin embargo, no se pudo determinar la causa que generó este comportamiento, debido a que no se encontraron evidencias de los mecanismos que generan altos niveles de DP.

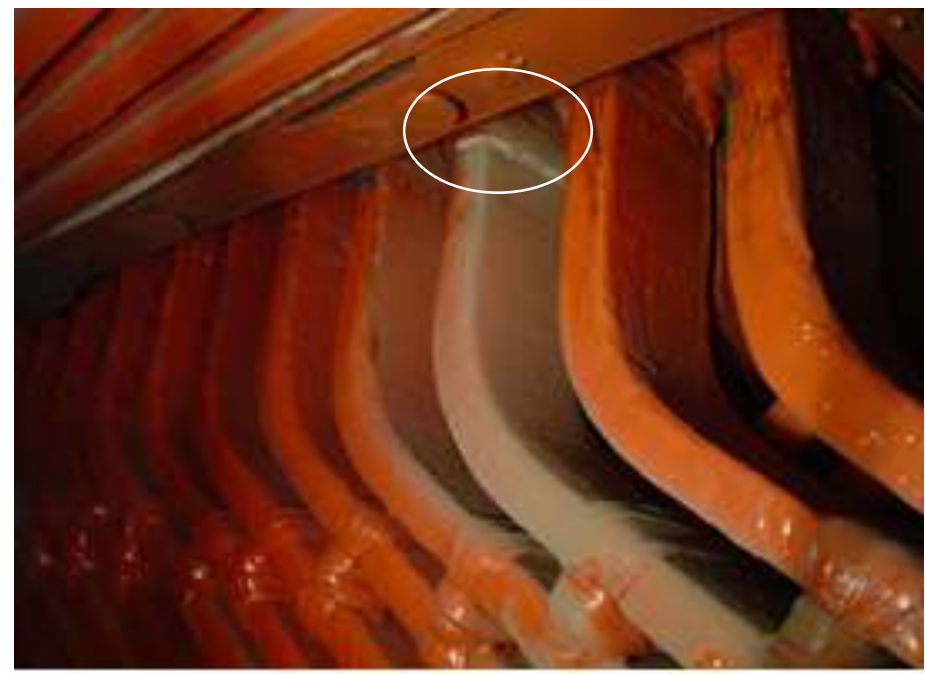

Fig. 3. Polvo blanco producto de la actividad de descargas parciales por problemas de graduación de campo eléctrico.

Para determinar la causa que generó los pulsos esporádicos de DP, el sobrecalentamiento sufrido por el generador bajo estudio, fue simulado en el laboratorio, utilizando bobinas de repuesto. Posteriormente, se llevó a cabo el seguimiento del mecanismo de deterioro, mediante el monitoreo del generador con pruebas dieléctricas fuera de línea. La descripción del mecanismo de deterioro, así como su seguimiento a través del tiempo, pueden constituir una referencia importante, para aquellos ingenieros que puedan enfrentar un problema similar, donde se requiera analizar un generador que ha sufrido un sobrecalentamiento, por problemas en su sistema de enfriamiento.

\section{EVALUACIÓN DIELÉCTRICA DEL GENERADOR}

El generador bajo estudio fue evaluado fuera de línea, con los métodos normalizados para la medición de DP, DP a la ranura y tan $\delta$ (IEEE 1434, 2014; IEEE 286, 2000). En la Fig. 4 se presentan solamente los valores registrados en la Fase $A$, para simplificar el análisis. En la Fig. 4, se muestra que la actividad de DP inició a $3.5 \mathrm{kV}$. Sin embargo, a $5.0 \mathrm{kV}$ aparecieron pulsos esporádicos de $20 \mathrm{nC}$, los cuales aparecían y desaparecían aleatoriamente en la pantalla del equipo de medición. A $6 \mathrm{kV}$, los pulsos esporádicos se incrementaron de 20 a 40 nC. Debido a que los pulsos de DP aparecían y desaparecían aleatoriamente, no se logró determinar si los pulsos positivos eran mayores que los negativos o viceversa, como lo indican los 
patrones de DP ocasionados por la separación del aislamiento del conductor y por DP a la ranura respectivamente (Hudon y Bélec, 2005). En la Fig. 4, también se observa que el $\Delta \tan \delta$, de 2.0 a $10.0 \mathrm{kV}$, fue menor del $1 \%$. Un criterio de diagnóstico reportado en la literatura, indica que un devanado que presenta un $\Delta \tan \delta$ menor del $2 \%$, de 2 a $10 \mathrm{kV}$, se puede considerar en buenas condiciones dieléctricas, con un bajo contenido de cavidades internas (CRIEPI, 1991). Este comportamiento descartó la posibilidad de que el sobrecalentamiento hubiese ocasionado la delaminación generalizada de las capas aislantes del generador bajo estudio.

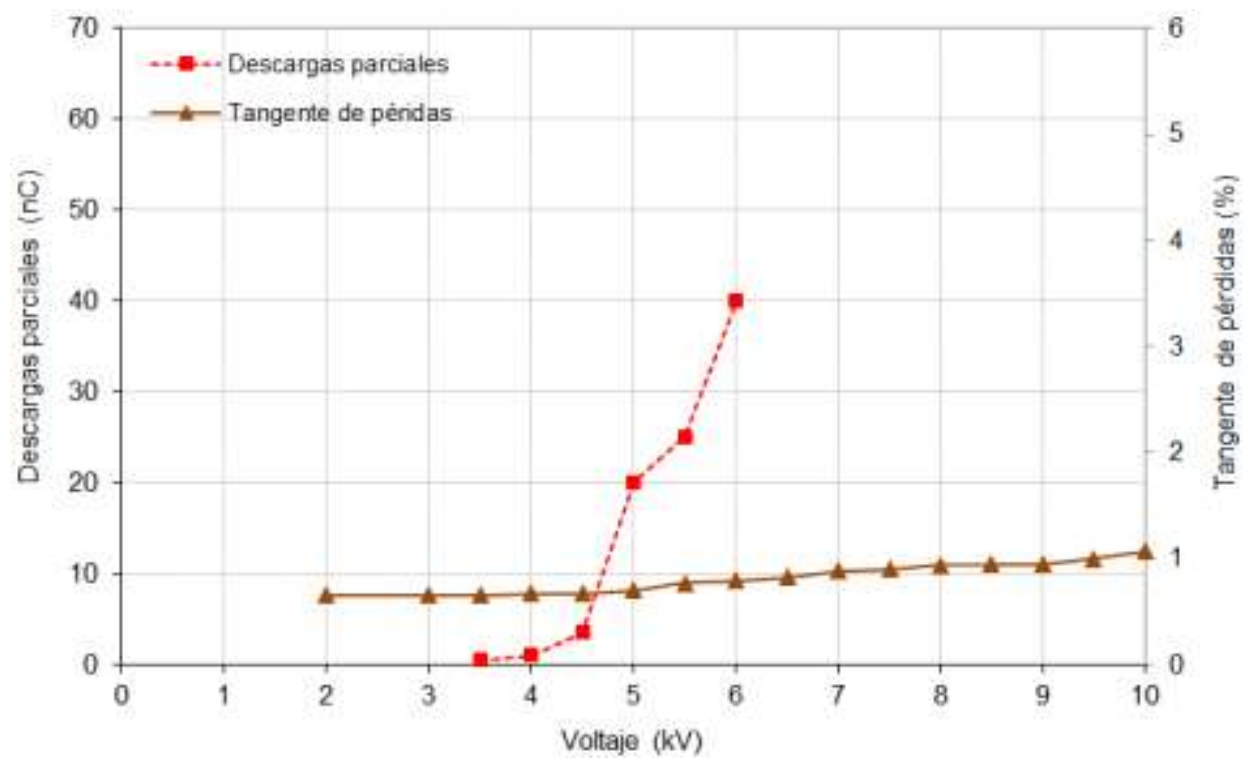

Fig. 4 Descargas parciales y tangentes de pérdidas en los devanados del generador inmediatamente después de su sobrecalentamiento.

Con la medición de descargas a la ranura, utilizando un sensor electromagnético sincronizado a $5 \mathrm{MHz}$, se registraron los niveles de ionización de cada una de las ranuras del estator a $5.5 \mathrm{kV}$, tal como se muestra en la Fig. 5. La Norma IEEE 1434, indica que los niveles mayores de $20 \mathrm{~mA}$, son generados por DP a la ranura (IEEE 1434, 2014). En este caso, se retiraron las cuñas de 10 de las 17 ranuras con los más altos niveles de ionización (de 20 a $30 \mathrm{~mA}$ ). Sin embargo, no se encontraron evidencias de deterioro en la pintura conductora de las bobinas, ocasionado por DP a la ranura y/o por abrasión de la pintura conductora. Por otro lado, la resistencia superficial de la pintura conductora se encontró con valores dentro del intervalo recomendado de 0.1 a $10 \mathrm{k} \Omega$ por cuadro (Azizi y Gholami, 2014). Finalmente, los devanados del generador, fueron energizados en la oscuridad, con $8 \mathrm{kV}$ de tensión de prueba. En estas condiciones, la actividad de DP fue audible pero no visible, por lo que se descartó la posibilidad de problemas de graduación de campo eléctrico en los devanados.

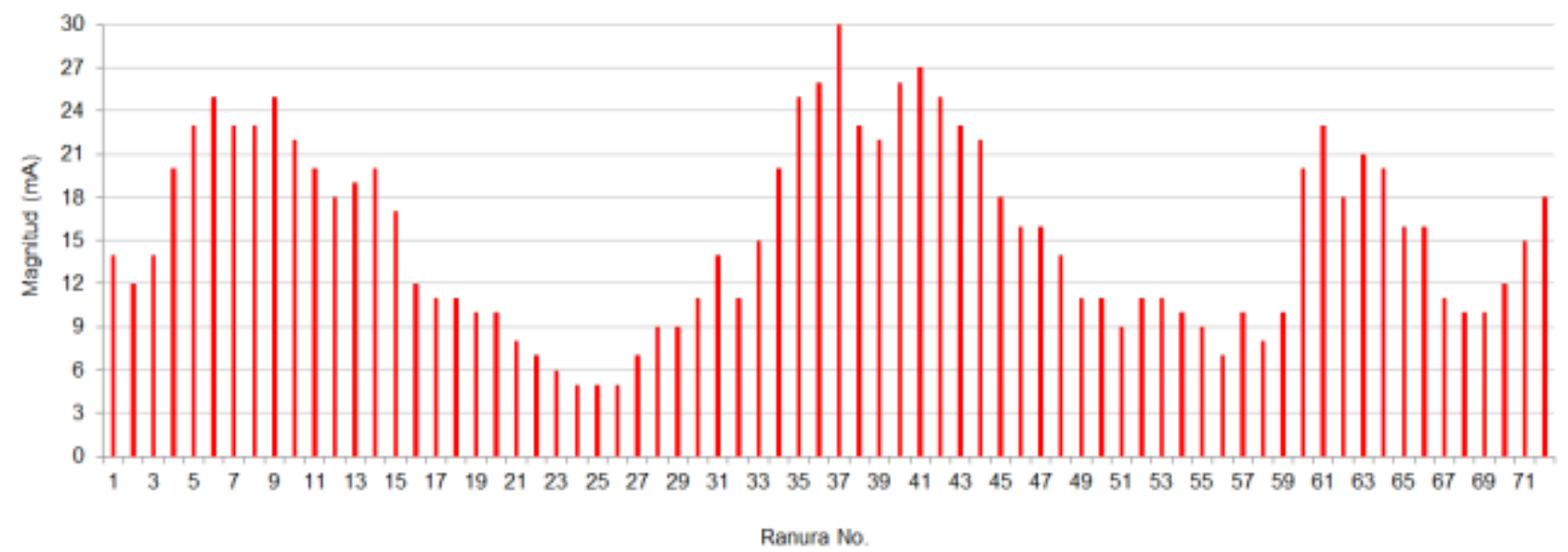

Fig. 5 Magnitud de descargas parciales en las ranuras del generador que sufrió el sobrecalentamiento (Voltaje de prueba $5.5 \mathrm{kV}$ ). 


\section{IDENTIFICACIÓN DEL MECANISMO DE DETERIORO}

Para identificar el mecanismo de deterioro del generador bajo estudio, el sobrecalentamiento fue simulado en el laboratorio. Se utilizaron dos bobinas de repuesto del propio generador, las cuales fueron seccionadas para obtener 4 muestras de $0.5 \mathrm{~m}$ de longitud. Una muestra fue utilizada para determinar el nivel de corriente que debería circular en el conductor de la bobina, para obtener $150{ }^{\circ} \mathrm{C}$, en la superficie de la pintura conductora. Las tres muestras restantes fueron acondicionadas con guardas y con pintura graduadora, para las mediciones de $\tan \delta$ y DP. Una muestra fue sometida a un calentamiento similar al que sufrió el generador bajo estudio. Se utilizó la metodología indicada por la Norma IEEE 1310 (IEEE 1310, 2012). Se hizo circular corriente a través del conductor, para lograr una temperatura de $150{ }^{\circ} \mathrm{C}$, en la superficie de la pintura conductora. El calentamiento se aplicó durante $1 \mathrm{~h}$. El ciclo térmico fue evaluado con mediciones de DP y $\tan \delta$, con la medición de la resistencia superficial de la pintura conductora y con la disección de las muestras.

En la Fig. 6 se muestran los resultados obtenidos antes y después del calentamiento de $150{ }^{\circ} \mathrm{C}$, con las mediciones de DP y $\tan \delta$. Es importante señalar que los valores de $\tan \delta$ medidos en bobinas individuales, no pueden ser comparados con los valores medidos en devanados completos, debido a que en las bobinas se utilizan electrodos de guarda y en los devanados no (IEEE 286, 2000). Tampoco se pueden comparar los valores de DP medidos en las bobinas individuales con los valores medidos en los devanados completos, debido a que en devanados completos se pueden presentar problemas de superposición en los pulsos de descargas parciales, haciendo que el equipo de medición registre un valor mayor de DP. De acuerdo con esto, solo se compararon las tendencias de DP y $\tan \delta$, en función de la tensión de prueba.

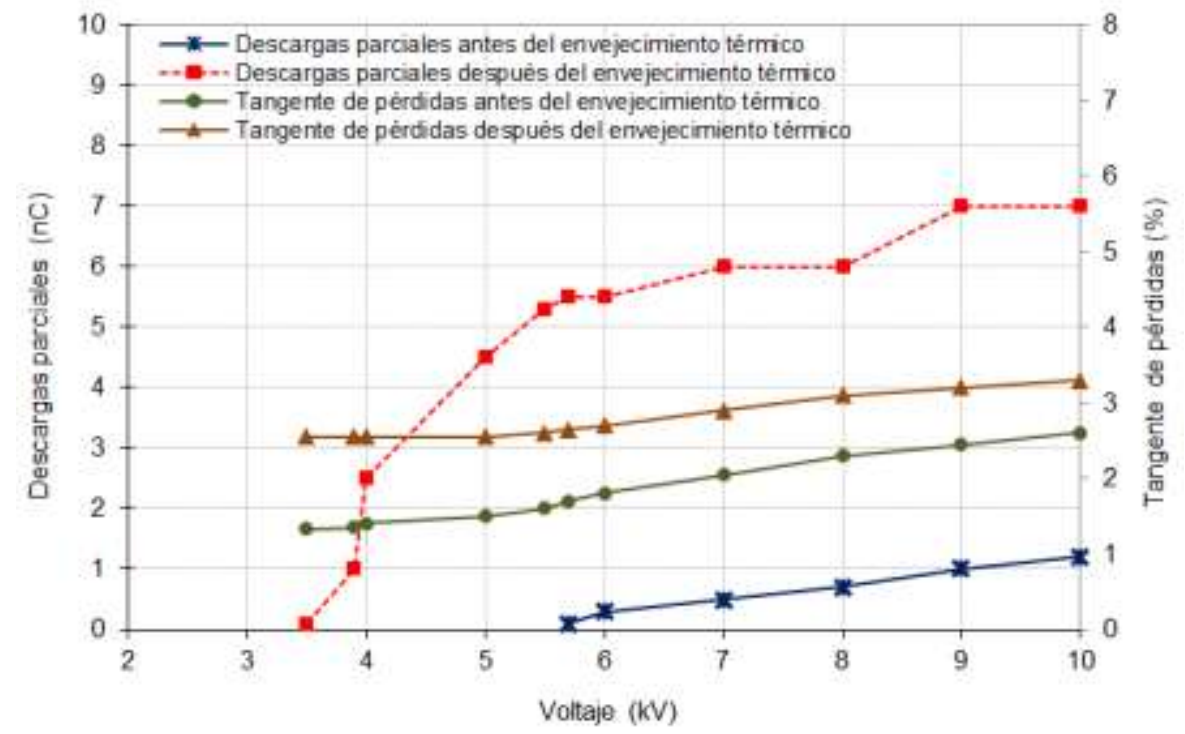

Fig. 6 Descargas parciales y tan $\delta$ en función del voltaje, antes y después del calentamiento, de una muestra a $150{ }^{\circ} \mathrm{C}$.

En la Fig. 6 se observa que, antes y después del calentamiento, el valor de $\Delta \tan \delta$ en cada paso de la tensión de prueba fue menor del $0.5 \%$. Esto indica que el aislamiento presentó un bajo contenido de cavidades internas antes y después del calentamiento (VDE 0530-1, 2011). Sin embargo, después del calentamiento, se presentó un cambio notable en la actividad de DP, con respecto al que se registró antes de éste. El voltaje de inicio de DP disminuyó de 5.8 a $3.5 \mathrm{kV}$. A $5 \mathrm{kV}$, aparecieron pulsos esporádicos de $\mathrm{DP}$, tal como sucedió en el generador bajo estudio. A $10 \mathrm{kV}$, los pulsos esporádicos fueron de $7 \mathrm{nC}$. En la muestra se escuchó una ionización intensa, pero no se observaron DP externas, por lo que se consideró que las DP estaban ocurriendo internamente en el sistema aislante.

Con la finalidad de analizar los posibles cambios internos en el aislamiento, se hizo un corte transversal en la muestra sometida al calentamiento de $150^{\circ} \mathrm{C}$ y en una muestra que no fue sometida a dicho calentamiento. Posteriormente, se tomaron imágenes amplificadas de las capas aislantes, tal como se muestra en las Figuras 7.a y 7.b. En estas figuras, se observa que no hay diferencias significativas en la estructura interna del aislamiento de la muestra que fue sometida al calentamiento con respecto a la muestra que no fue sometida a dicho calentamiento. Esto indica que el calentamiento no ocasionó la separación entre el aislamiento y el conductor y tampoco generó una delaminación generalizada entre las capas aislantes. Por esta razón, la curva de tan $\delta$ vs voltaje, presentó incrementos reducidos tanto en el generador bajo estudio, como en la muestra, después del sobrecalentamiento de $150^{\circ} \mathrm{C}$. 
La delaminación del sistema aislante, se logró cuando la tercera muestra fue sometida a un calentamiento hasta de $160{ }^{\circ} \mathrm{C}$, medidos en la superficie de la pintura conductora, durante 1 hora. Al seccionar la muestra y amplificar la imagen del aislamiento, se observó la separación de las capas aislantes, cerca del conductor. Esta separación se muestra encerrada por una elipse de color blanco, en la Fig. 7.c.

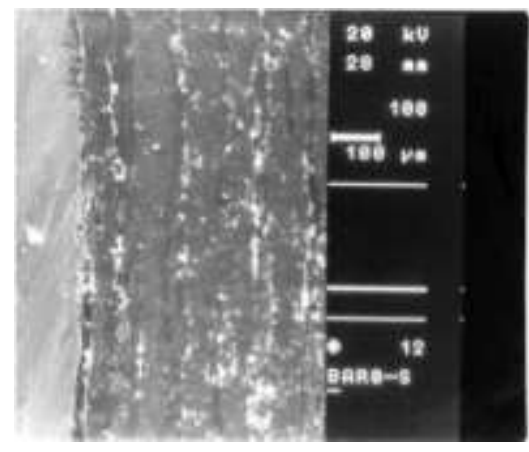

(a)

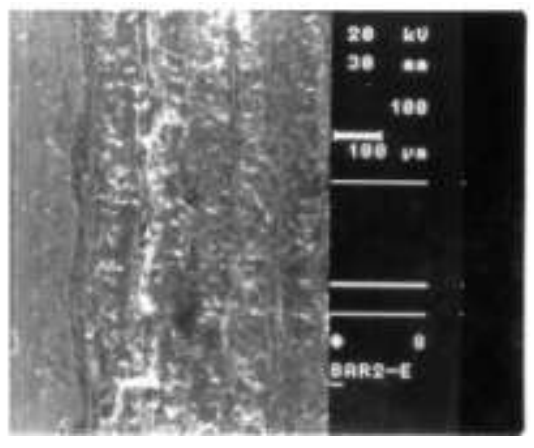

(b)

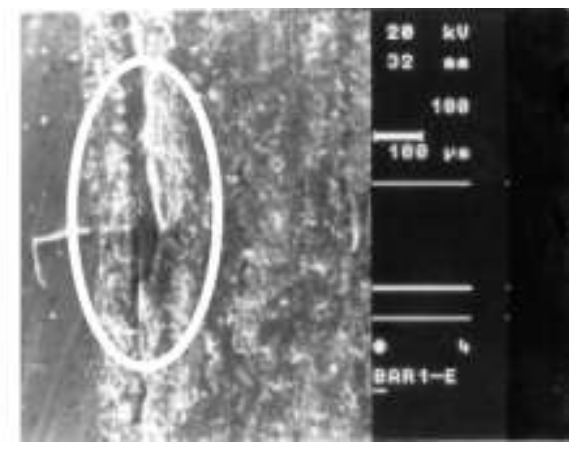

(c)

Fig. 7 Imágenes amplificadas 100 veces del sistema aislante de las muestras. (a) Muestra sin calentamiento. (b) Muestra después del calentamiento a $150^{\circ} \mathrm{C}$. (c) Muestra después del calentamiento a $160^{\circ} \mathrm{C}$.

En la muestra que fue calentada a $150^{\circ} \mathrm{C}$, la resistencia superficial de la pintura conductora, no presentó cambios significativos después del calentamiento, manteniendo su valor dentro del intervalo recomendado de 0.1 a $10 \mathrm{k} \Omega$ por cuadro (Azizi y Gholami, 2014). Sin embargo, se observó que en algunas zonas, la pintura conductora se separaba de la superficie del aislamiento, en forma de láminas quebradizas. La Norma IEEE 1310 indica que un aislamiento Clase $\mathrm{F}$ debe soportar 500 ciclos térmicos de $150{ }^{\circ} \mathrm{C}$ (IEEE $1310,2012)$. En el generador bajo estudio, el sistema aislante soportó el sobrecalentamiento equivalente a un ciclo térmico correspondiente al aislamiento Clase F. Sin embargo, la pintura conductora no soportó este esfuerzo térmico, indicando que no cumplía con los requerimientos de la Clase $\mathrm{F}$. Como consecuencia, la pintura se separó de la superficie del aislamiento, en algunas zonas. De acuerdo con esto, se infirió que las cavidades internas que se formaron debajo de la pintura conductora, fueron las que se ionizaron con la tensión de prueba, ocasionando los pulsos esporádicos de DP de gran magnitud.

La formación de cavidades internas, debajo de la pintura conductora, ya fue reportada en la literatura especializada (Stone, et al, 2009). En el caso publicado, las cavidades internas debajo de la pintura conductora, se atribuyeron a una pobre calidad en la manufactura de la pintura, en un grupo de máquinas fabricadas en la década de los 70's. En México, en la década de los 70's, los generadores todavía no eran evaluados con mediciones de DP y tan $\delta$, ni se realizaban pruebas con ciclos térmicos a bobinas prototipo. Por esta razón, la deficiencia en la clase térmica de la pintura conductora del generador bajo estudio, no pudo ser detectada al inició de su operación comercial.

Es importante señalar, que el generador bajo estudio operaba a su capacidad nominal, con una temperatura del orden de $90^{\circ} \mathrm{C}$, en la superficie de sus devanados. Por otro lado, los umbrales de protección por sobre temperatura se fijaban a 110 y $130^{\circ} \mathrm{C}$. Por lo tanto, el generador operó durante 18 años con esfuerzos térmicos que no alcanzaron ni la temperatura de la Clase $B\left(130^{\circ} \mathrm{C}\right)$, por lo que térmicamente operó con un amplio margen de seguridad, sin que la pintura conductora sufriera algún daño.

\section{SEGUIMIENTO DEL MECANISMO DE DETERIORO}

Se consideró que para obtener una operación confiable, la pintura conductora de las bobinas del generador, tenía que ser sustituida, por una pintura Clase F. Sin embargo, por requerimientos de la planta, el generador bajo estudio tuvo que continuar en operación. Esta situación se aprovechó para dar seguimiento al mecanismo de deterioro, a través del tiempo. Durante 12 años se realizaron mediciones de DP y tan $\delta$ fuera de línea, con intervalos de medición de 4 años aproximadamente. Para simplificar el análisis, en las Figuras 8 y 9 se muestran los resultados obtenidos solamente en la Fase $A$.

En la Fig. 8 se observa que la gráfica de tan $\delta$, prácticamente no cambió después de 4 y 8 años de servicio, con respecto a la curva obtenida inmediatamente después del sobrecalentamiento. De 2.0 a $10.0 \mathrm{kV}$ el $\Delta \tan$ $\delta$ se mantuvo con un valor menor del $1 \%$. Este resultado confirma que el sobrecalentamiento de $150{ }^{\circ} \mathrm{C}$ no ocasionó la delaminación del aislamiento (CRIEPI, 1991). 


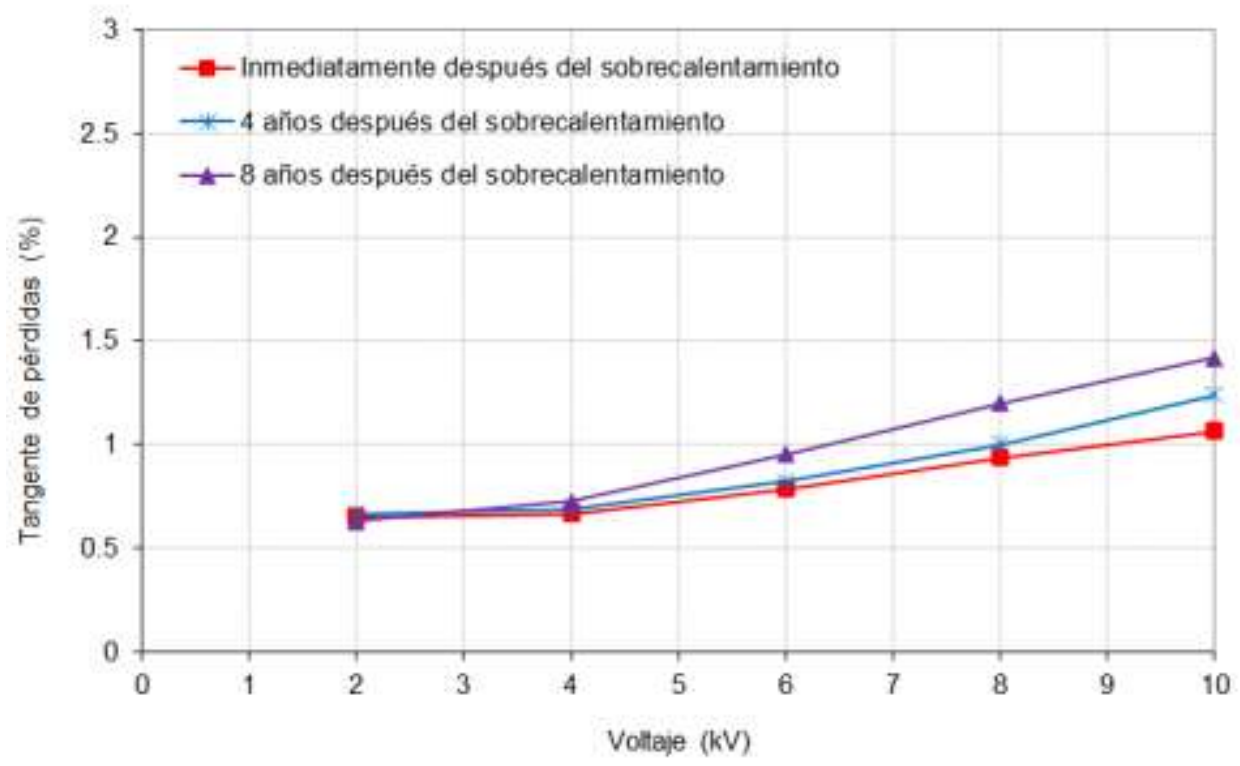

Fig. 8 Tan $\delta$ en función del tiempo, en el generador que sufrió un sobrecalentamiento.

En la Fig. 9, se observa que después de 4 años de servicio, la gráfica de $D P$, de 3.0 a $5.5 \mathrm{kV}$ fue prácticamente la misma que se obtuvo inmediatamente después del sobrecalentamiento. Sin embargo, los pulsos esporádicos de DP se convirtieron en pulsos que aparecían permanentemente. Esto permitió identificar que los pulsos negativos eran mayores a los pulsos positivos, tal como corresponde al patrón generado por el fenómeno de DP a la ranura (Hudon y Bélec, 2005).

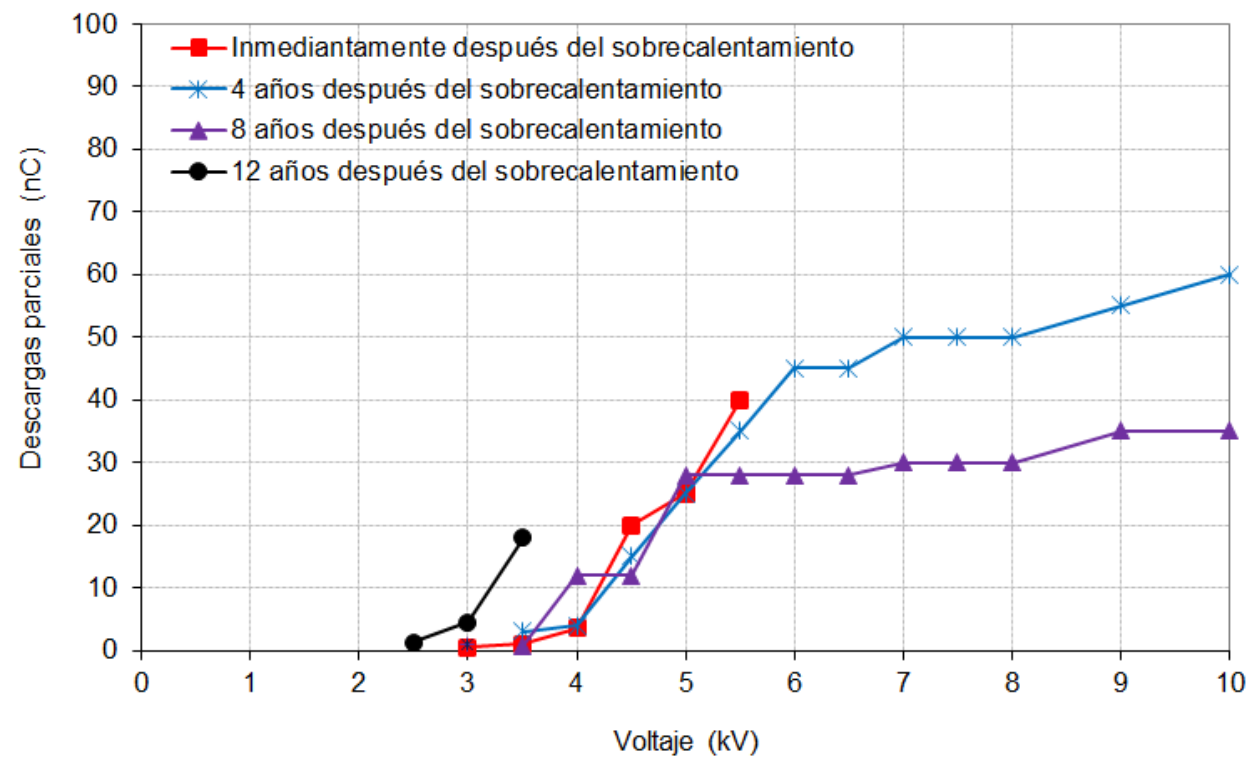

Fig. 9 Descargas parciales en función del tiempo, en el generador que sufrió un sobrecalentamiento.

Después de los 8 años de servicio, la gráfica de DP vs tensión de prueba, se desplazó hacia abajo con respecto a la curva obtenida a los 4 años del deterioro. Por ejemplo a $6.0 \mathrm{kV}$, las DP disminuyeron de 45 a menos de $30 \mathrm{nC}$. A los $10 \mathrm{kV}$, las DP disminuyeron de 60 a $35 \mathrm{nC}$. El patrón de DP continuó siendo asimétrico, generado por DP a la ranura.

A los 12 años de operación, la gráfica de DP vs tensión se desplazó hacia la izquierda con respecto a las gráficas anteriores. Esto indicó que las DP aparecían a una tensión de prueba menor. Los pulsos del orden de $20 \mathrm{nC}$, que aparecía a $4.5 \mathrm{kV}$, ahora aparecieron a $3.5 \mathrm{kV}$. Finalmente, al incrementar la tensión de 3.5 a $4.0 \mathrm{kV}$ se presentó una falla a tierra. Como la falla ocurrió a $4 \mathrm{kV}$, se consideró que la bobina que falló, operaba a una tensión inferior a 4 kV. De lo contrario, hubiera fallado antes, en operación. 


\section{CIRCUITO EQUIVALENTE Y TASA DE DETERIORO DEL MECANISMO DETECTADO}

Con la finalidad de comprender el comportamiento de la actividad de DP en el generador bajo estudio, a través de los años de servicio, se consideró el circuito equivalente de una bobina en una ranura del estator, que se muestra en la Fig. 10. En condiciones normales de operación, Fig. 10 (a), la resistencia Rp está conectada entre $\mathrm{Ca}$ y el núcleo a potencial de tierra, por lo que el campo eléctrico queda confinado en el aislamiento principal. En estas condiciones, si se presentan DP, serían del tipo interno.
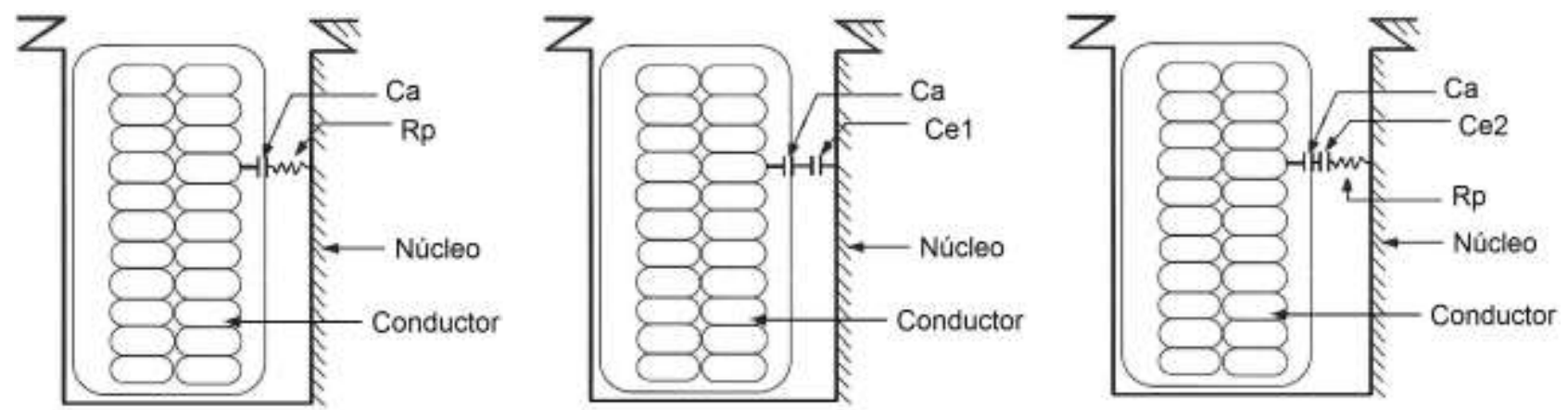

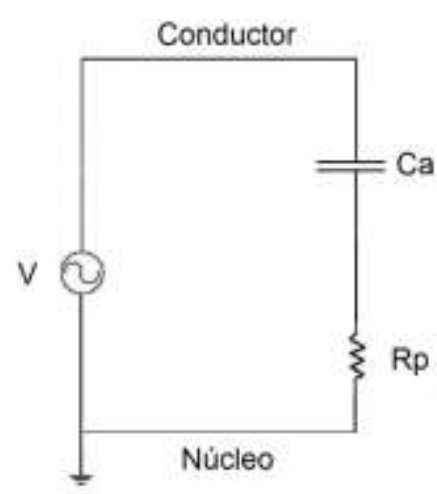

(a)

Ca: capacitancia del aislamiento Rp: resistencia superficial de la pintura conductora

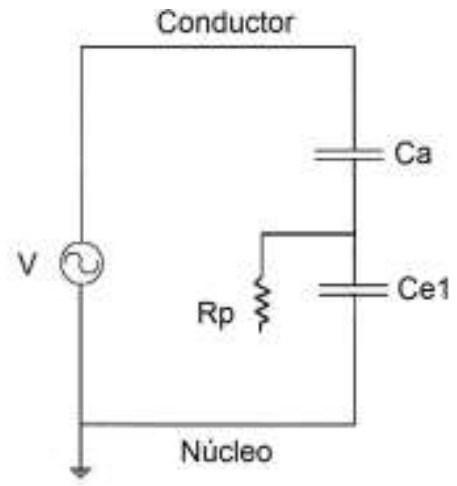

(b)

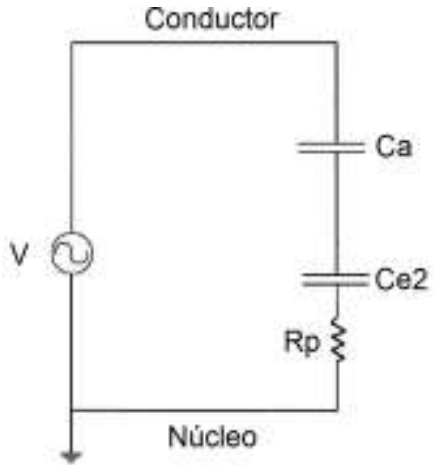

(c)

Ce1: capacitancia del espacio con aire entre la superficie de la bobina y el núcleo Ce2: capacitancia del espacio con aire entre la superficie de la bobina y la pintura conductora

Fig. 10: Circuito equivalente de una bobina en la ranura del estator. (a) Arreglo en condiciones normales de operación. (b) Superficie de la bobina con pérdida de la pintura conductora. (c) Cavidades microscópicas debajo de la pintura conductora

Cuando la pintura conductora deja de hacer contacto con las laminaciones del núcleo del estator, el circuito equivalente cambia al arreglo de la Fig. 10 (b), donde la resistencia Rp queda desconectada. Ahora se tiene un divisor capacitivo en donde, el gradiente eléctrico será mayor en Ce1 por la diferencia de permitividades entre el aislamiento y el aire. Se pueden alcanzar gradientes superiores a $3 \mathrm{kV} / \mathrm{mm}$ en Ce1, en gran parte del devanado, dependiendo de la distancia entre la superficie de la bobina y la laminación del núcleo, generando DP a la ranura. Esto indica que las DP a la ranura pueden ocurrir en todas las bobinas, independientemente de su tensión de operación.

El mecanismo de deterioro que se presenta en este artículo, ocurrió cuando la pintura conductora se separó de la superficie de las bobinas, por lo que el circuito equivalente resultante se muestra en la Fig. 10 (c). En este arreglo, también se tiene un divisor capacitivo. Sin embargo, ahora las DP se presentan en la capacitancia $\mathrm{Ce} 2$, formada por la cavidad de aire entre la pintura y la superficie de la bobina y no entre la pintura y las laminaciones del núcleo. La tasa de deterioro de las DP es función de la energía involucrada y queda determinada por la ecuación siguiente:

$E=1 / 2 C V^{2}$

Dónde $\mathrm{E}$ es la energía de las descargas, $\mathrm{C}$ es la capacitancia de la bobina durante la descarga y $\mathrm{V}$ es el voltaje de operación de la bobina. 
En la ecuación (1) se observa, que la energía de las descargas depende de la capacitancia que se está descargando y del cuadrado de la tensión de operación de las bobinas. En la primera etapa del fenómeno, la energía es alta porque la capacitancia que se descarga, está formada prácticamente por toda la zona cubierta con la pintura conductora. Esto explica los niveles altos de DP esporádicos, que se registraron en el generador bajo estudio, inmediatamente después del sobrecalentamiento y en los primeros cuatro años de servicio, posteriores al sobrecalentamiento.

Conforme la pintura conductora se va erosionando, por la acción de las DP, la capacitancia que se descarga va disminuyendo en magnitud. Por lo tanto, la intensidad y la energía de las descargas también disminuyen, de acuerdo con la ecuación (1). Esto explica lo ocurrido en la segunda etapa del mecanismo de deterioro del generador bajo estudio. Las DP que ocurrían debajo de la pintura conductora, fueron erosionaron la propia pintura, hasta dejar zonas eléctricamente "flotadas" en la superficie de las bobinas. Al disminuir la capacitancia que se estaba descargando, la energía, la magnitud y la tasa de deterioro de las DP también disminuyeron, después de 8 años de servicio. Una vez que la pintura ha sido erosionada, las DP se concentran entre las zonas eléctricamente "flotadas" de las bobinas afectadas y las laminaciones del núcleo. En consecuencia, las DP comienzan a atacar y erosionar una zona muy puntual del aislamiento. Este comportamiento ocasionó, que a los 12 años de servicio, se presentara una falla a tierra, en el generador bajo estudio.

Debido al término cuadrático en la ecuación (1) la energía de las descargas es función directa de la posición eléctrica de la bobina en el devanado. De esta forma, las bobinas cercanas al inicio de línea sufren un deterioro mayor que las bobinas cercanas al neutro. Este dato es significativo porque la bobina que falló estaba localizada eléctricamente en la primera mitad del devanado. Puede concluirse que dependiendo de la posición eléctrica de las bobinas afectadas, dependerá también el tiempo de vida útil de las bobinas de acuerdo con la ecuación (1). En este caso en específico en que un devanado sufrió un sobrecalentamiento, una de las bobinas afectadas y que estaba localizada en la parte media del devanado tardó doce años en fallar.

\section{CONCLUSIONES}

Se analizó el mecanismo de deterioro de un generador que sufrió un sobrecalentamiento de $150{ }^{\circ} \mathrm{C}$. Del sistema aislante de las bobinas del estator, la pintura conductora fue la que no cumplió térmicamente con la Clase F, por lo que perdió adhesión, separándose de la superficie del aislamiento de las bobinas, en algunas zonas del devanado. Esto generó cavidades internas debajo de la pintura, lo cual originó pulsos esporádicos de descargas parciales de gran magnitud.

Con evaluaciones programadas fuera de línea en el generador, se observó que los pulsos esporádicos de DP que se detectaron inmediatamente después del calentamiento se convirtieron en pulsos permanentes, con una configuración típica de descargas a la ranura en la cual los pulsos negativos eran mayores a los pulsos positivos.

Se identificaron dos etapas en el deterioro del generador. La primera involucró descargas parciales de gran magnitud, que erosionaron la pintura conductora hasta dejar zonas eléctricamente "flotadas" en la superficie de las bobinas. En la segunda etapa, en las zonas de las bobinas que quedaron eléctricamente "flotadas, el nivel de descargas parciales disminuyó pero se concentró en zonas muy puntuales del aislamiento hasta ocasionar el rompimiento dieléctrico. En el caso que se reporta, el proceso de erosión tardó 12 años en ocasionar una falla a tierra en una bobina localizada eléctricamente en la primera mitad del devanado.

\section{REFERENCIAS}

Azizi, D. y Gholami, A., Optimization of Semiconductive Coating and Groundwall Insulation Layers in Stator Slot of HV Generator, doi: 10.1016/j.ijepes.2013.12.018, Elsevier, Electrical Power and Energy Systems 57, 384-391 (2014)

Azuaje, C. y Torres, W., Experiences in Identification of Partial Discharge Patterns in Large Hydrogenerators, CIGRE A1-2009, session (2004)

Campuzano, I.R., Diagnóstico de Generadores Eléctricos de Potencia con Técnicas de Monitoreo en Línea y Fuera de Línea, Inf. Tecnol. 27(2), en prensa (2016)

Central Research Institute of Electric Power Industry, An Insulation Deterioration Diagnostic Method for Generator Windings, Special Document for IERE members (R-99019), Japan, (1991) 
Darwanto, D., Hamdani, D., Hariyanto, D. y Karyawan, H., Partial Discharge Analysis for Predictive Maintenance of Generator of Geothermal Power Plant, doi: 10.1109/CMD.2012.6416252, International Conference on Condition Monitoring and Diagnosis (CMD), 23-27 September 2012, 740-743 (2012)

Dorrbercker, S., Torres, W. y Molé, J., Experiencias en el Diagnóstico del Aislamiento de los Generadores de Potencia. http:www://scielo.sld.cu/scielo.php?script=sci_arttext\&pid=S18159012012000100007, ISSN 1815-5901 Ingeniería Energética, Vol. XXXIII, No. 1/ enero-abril 2012, 59-67(2012)

Hudon, C. y Belec, M., Partial Discharge Signal Interpretation for Generator Diagnostics, IEEE Transactions on Dielectrics and Electrical Insulation, Vol. 12, No. 2, ISSN 1070-9878, 297- 319 (2005)

IEEE Std 286, Recommended Practice for Measurement of Power Factor Tip-Up of Electric Machinery Stator Coil Insulation, (2000)

IEEE Std 1310, Recommended Practice for Thermal Cycle Testing of Form-Wound Stator Bars and Coils for Large Rotating Machines, (2012)

IEEE Std 143,. Trial-Use Guide to the Measurement of Partial Discharges in Rotating Machinery, (2014)

Krpal, O. y Mráz, P., V-A Characteristic Measuring of Stress Grading Tapes in the End-Winding of Synchronous Generators, doi: 10.1016/j.proeng.2014.03.150, Procedia Engineering 69 ( 2014 ), Published by Elsevier, ScienceDirect, 24th DAAAM International Symposium on Intelligent Manufacturing and Automation, 1523 - 1528 (2013)

Sathiyasekar, K., Thyagarajah, K., Krishnan, A. y Jeyakumar A., Condition Monitoring of Stator Winding Insulation in High Voltage Rotating Machines Using BPN Algorithm, International Journal of Electrical and Power Engineering, Volume: 3, Issue: 2, Page No.119-123 (2009)

Stone, G., Boulter, E. A., Culbert, I.C. y Hussein D., Electrical Insulation for Rotating Machines: Design, Evaluation, Aging, Testing and Repair, $2^{\text {nd }}$ Edition, 152-153, IEEE Press Series on Power Engineering Mohamed E. El-Hawary, Series Editor (2004)

Stone, G., Maughan, C., Nelson, D. y Schultz, R., Impact of Slot Discharges and Vibration Sparking on Stator Winding Life in Large Generators, IEEE Electrical Insulation Magazine, September/October, Vol. 24, No. 5, (2008)

Stone, G., Sasic, M., Dunn, D. y Culbert, I.C. Recent Problems Experienced with Motor and Generator Windings. doi: 10.1109/pcicon.2009.5297173, Petroleum and Chemical Industry Conference, PCIC 2009, 2009 Record of Conference Papers - Industry Applications Society 56th Annual. (2009)

Torres, W., Dorrbercker, S. y Benitez I., Variable para la Supervisión del Envejecimiento del Aislamiento Principal de Grandes Hidrogeneradores Mediante Descargas Parciales, ISSN 1316-4821, Universidad, Ciencia y Tecnología, Volumen 14, No. 56, pp 165-173 septiembre (2010)

VDE 0530-1, Standard Specification for Rotating Electrical Machines, Part 1, (2011)

Znidarich, M., Hydro Generator High Voltage Stator Windings: Part 1 - Essential Characteristics and Degradation Mechanisms, doi:10.1080/1448837X.2008.11464196, Australian Journal of Electrical and Electronics Engineering, Volume 5, Issue 1, 1-17 (2008) 